\title{
ELECTRICAL RESISTIVITY AND POSITRON LIFETIME STUDIES OF PRECIPITATION EFFECTS IN Al-Cu ALLOY
}

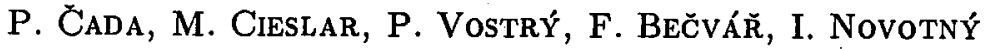 \\ AND I. ProcházKa
}

Faculty of Mathematics and Physics, Charles University

Ke Karlovu 3, 12116 Prague 2, Czech Republic

\begin{abstract}
Decomposition of the supersaturated solid solution of 2 at.\% $\mathrm{Cu}$ in Al was investigated by means of electrical resistivity and high-resolution positron lifetime measurements. The phase composition of this alloy was determined by transmission electron microscopy. Electrical resistivity measurements were performed by a classical four-point method. Positron lifetime spectra were measured by means of a spectrometer consisting of two $\mathrm{BaF}_{2}$ detectors and a standard fast-slow coincidence system. The specimens were first exposed to the solution heat treatment at $783 \mathrm{~K}$ for 19 hours with a subsequent quenching. Then the specimens were isochronally annealed in the temperature range $293 \div 573 \mathrm{~K}$. Annealing responses of electrical resistivity and positron annihilation were studied. Resistivity measurements as well as transmission electron microscopy observations confirmed the well-known decomposition sequence of the $\mathrm{Al}-\mathrm{Cu}$ alloy. The decomposition of the alloy was manifested by the decrease in the intensity of positron lifetime component $\tau_{2}=(207 \pm 2)$ ps correlated with simultaneous appearance and increase in the intensity of $\tau_{3}=(180 \pm 5)$ ps component. Component $\tau_{2}$ originates from positron annihilation in vacancies trapped at the Guinier-Preston zones while $\tau_{3}$ comes from annihilation of the positrons localized in the misfit dislocations at coherent precipitates of the $\mathrm{Al}_{2} \mathrm{Cu}$ phase. The shortest observed component $\tau_{1}$ apparently belongs to annihilation of untrapped positrons.
\end{abstract}

PACS numbers: 61.72. $-\mathrm{y}, 78.70 .-\mathrm{g}, 81.40 .-\mathrm{z}$

\section{Introduction}

Binary alloy $\mathrm{Al}-\mathrm{Cu}$ belongs to commonly used hardenable materials. Moreover, many important technical alloys are based on this system.

The decomposition of the supersaturated solid solution of $\mathrm{Cu}$ in $\mathrm{Al}$ is well described in the literature [1]. At low concentration of $\mathrm{Cu}$ and below $573 \mathrm{~K}$ it occurs in the sequence: creation of monolayer and multilayer Guinier-Preston (GP) zones 
$\rightarrow$ coarsening of the GP zones and their partial arrangement in $(110\rangle$ directions

$\rightarrow$ transformation of the GP zones into coherent particles of the $\mathrm{Al}_{2} \mathrm{Cu}$ phase. Nevertheless, some details of this process remain unclear and their investigation by means of modern physical methods is still in progress [2].

The present paper deals with a detailed study of the decomposition of the supersaturated solid solution of 2 at.\% $\mathrm{Cu}$ in $\mathrm{Al}$ using three experimental methods: electrical resistometry, electron microscopy and positron lifetime technique. The high-resolution positron lifetime measurements were applied with the hope to obtain supplementary information about the role of intrinsic crystal lattice defects and to detect separately individual overlapping phase transformation processes. In order to reach these goals, a positron lifetime spectrometer [3] with superior timing resolution power of $\approx 150 \mathrm{ps}$ (FWHM) for ${ }^{22} \mathrm{Na}$ was used.

\section{Experimental details}

\subsection{Specimens}

The investigated alloy $\mathrm{Al}-2$ at.\% $\mathrm{Cu}$ was prepared from $\mathrm{Al} 99.9995$ at.\% and $\mathrm{Cu} 99.995$ at.\% in Research Institute of Metals, Panenské Břežany. The resulting concentration of $\mathrm{Cu}$ in the alloy was determined as 2.05 at.\% (4.7 wt.\%) by the mass spectroscopy and chemical analysis. The polycrystalline specimens for positron annihilation, electrical resistometry and electron microscopy were prepared from the sheet of the $1 \mathrm{~mm}$ thickness. The final form of specimens was determined by requirements of the experimental method applied.

\subsection{Positron lifetime measurements}

The detector part of the spectrometer [3] consists of two $\mathrm{BaF}_{2}$ cylindric crystals (Korth Kristalle GmbH) coupled to the photomultiplier tube (PMT) Philips XP 2020Q. Magnetic shielding of the photocathode is provided by means of a permalloy tube put on the duralumin housing. Special high-voltage distribution along the PMT dynode chain was chosen [3]. Timing and energy signals are taken from 10 th and 9 th dynode, respectively.

Output signals from detectors are processed by standard fast-slow coincidence system. The timing signal passes through a capacitor directly to the input of the constant-fraction discriminator (CFD). Timing signals from CFD's are led to the start and stop inputs of a time-to-amplitude converter (TAC). The energy signal from the detector is first shaped by a passive RC-integration circuit. Then the signal goes to a spectroscopy amplifier whose bipolar output pulses are led to a timing single-channel analyzer (TSCA) adjusted to appropriate $\gamma$-ray energy. Negative output pulses from TSCA's pass to the start and stop inputs of an auxiliary TAC serving as a coincidence circuit generating the strobe signal for the main $\mathrm{TAC}$ in the timing branch.

The output pulses from the main TAC are processed in an analog-to-digital converter whose data output is connected to a histogramming memory operated by an auxiliary CAMAC controller. The complete electronics is built from commercially available modules produced by ORTEC and CANBERRA (NIM) and by C.E.S. (CAMAC). 
The carrier-free ${ }^{22} \mathrm{NaCl}$ (Amersham) deposited and sealed between $2 \mu \mathrm{m}$ thick mylar foils is used as a positron source. A face-to-face detector geometry with common symmetry axis was chosen. The sandwich assembly of the positron source and the two discs of the investigated alloy (6.5 mm diameter, $1 \mathrm{~mm}$ thick) is located out of the common axis at a radial distance slightly greater than the radius of the scintillators. All measurements were performed at room temperature.

The spectrum was decomposed by a procedure [3] based on the maximum-likelihood principle assuming the Poisson distribution of the raw data. Model function was essentially the same as that of Ref. [4]. Optimum parameters were searched for using the MINUIT routine [5] on HP-715 workstation. Total FWHM of resolution function, composed of the three Gaussians, is $150 \pm 1 \mathrm{ps}$. The intensity ratio of the two dominating Gaussians and the time-shift of the weakest one were kept fixed during the model parameter fit.

\subsection{Electrical resistometry}

The specimens for electrical measurements were punched to the dimensions of $1 \times 5 \times 100 \mathrm{~mm}$ from the initial sheet. The current and potential contacts were prepared by cutting the strip. Electrical resistivity $\varrho$ was measured in liquid nitrogen by the standard DC four-point method with a dummy specimen in series. The measuring electrical circuit was powered from a supply Keithley $228 \mathrm{~A}$ and the data acquisition was performed by a personal computer. The potential drop across the specimen was read on a digital nanovoltmeter Keithley 181 while the measuring current $(2 \mathrm{~A})$ was monitored on a digital multimeter Keithley 195A with the use of normal resistance. The influence of parasite thermopowers was suppressed by the commutation of the measuring current. Each resistivity value was determined as the mean of ten measurements.

\subsection{Electron microscopy}

The foils for electron microscopy were prepared by the following procedure: electrical spark cutting, surface grinding and final electrolytic polishing (33\% $\left.\mathrm{HNO}_{3}+67 \% \mathrm{CH}_{3} \mathrm{OH}\right)$ in a twin-jet device TENUPOL. Transmission electron microscopy (TEM) and electron diffraction (ED) observations were performed on a microscope TESLA BS 540 operating at accelerating voltage of $120 \mathrm{kV}$.

\section{Results and discussion}

The specimens were first exposed to the solution heat treatment for 19 hours at $783 \mathrm{~K}$ finished by quenching into water and, after one minute, into liquid nitrogen. As a result we obtained a solid solution of $\mathrm{Cu}$ in $\mathrm{Al}$ with a certain number of fine GP zones. The solution treated specimens were isochronally annealed in a stepped programme based on the regime $10 \mathrm{~K} / 20 \mathrm{~min}$ and the annealing response of resistivity and positron lifetime were measured in the annealing temperature range $293 \div 573 \mathrm{~K}$. Following the literature (see e.g. [1]), the decomposition of supersaturated low-concentrated solution of $\mathrm{Cu}$ in $\mathrm{Al}$ occurs below $573 \mathrm{~K}$ in the sequence: creation of monolayer and multilayer GP zones $\rightarrow$ coarsening of the GP zones and their partial arrangement in $\langle 110\rangle$ directions $\rightarrow$ transformation of the GP zones into coherent particles of the $\Theta^{\prime}$-phase $\left(\mathrm{Al}_{2} \mathrm{Cu}\right)$. 


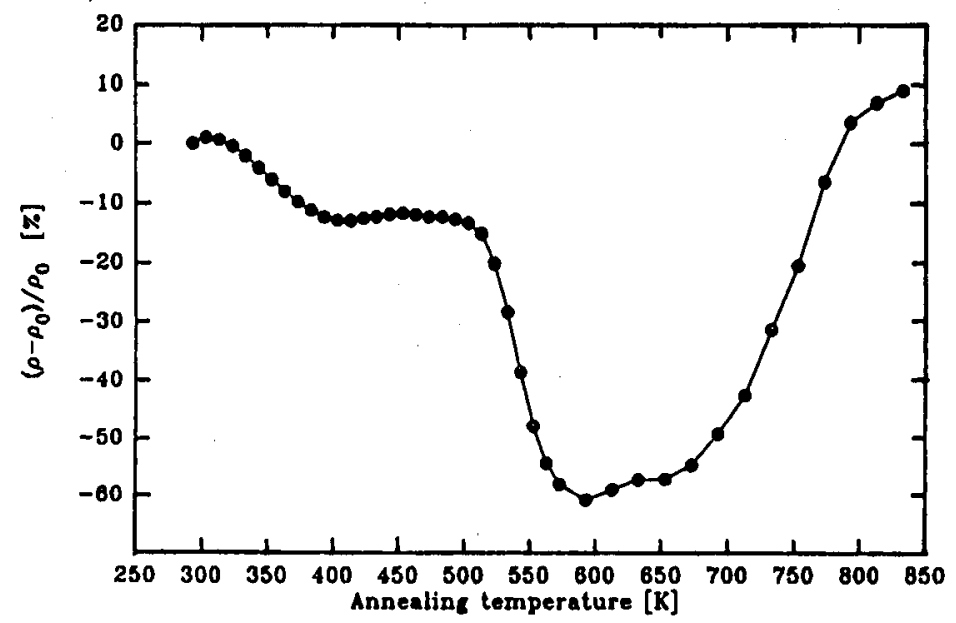

Fig. 1. Normalized isochronal annealing curve of electrical resistivity $\varrho$. The initial value of $\varrho$ in as solution treated specimen is denoted by $\varrho_{0}$.

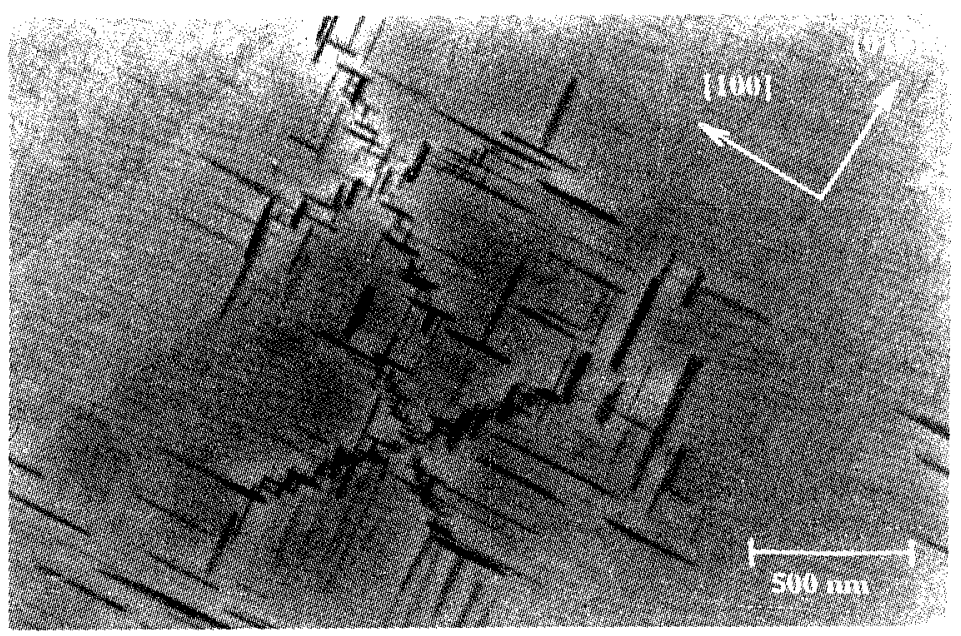

Fig. 2. TEM image of arrangement of the GP zones in the specimen isochronally annealed up to $443 \mathrm{~K}$.

The annealing response of resistivity (Fig. 1) reflects well the above mentioned sequence: the evolution of the GP zones' population $(290 \div 500 \mathrm{~K})$ and the transformation of large GP zones into the coherent precipitates $(500 \div 600 \mathrm{~K})$. We verified the decomposition sequence by TEM and ED (see Figs. 2 and 3). The increase in resistivity at higher temperatures (above $600 \mathrm{~K}$ ) is due to the redissolution of precipitates and reconstruction of solid solution of $\mathrm{Cu}$ in $\mathrm{Al}$.

In addition to the electrical resistivity studies, the positron lifetime measurements were carried out in the annealing temperature range of $323 \div 563 \mathrm{~K}$. 


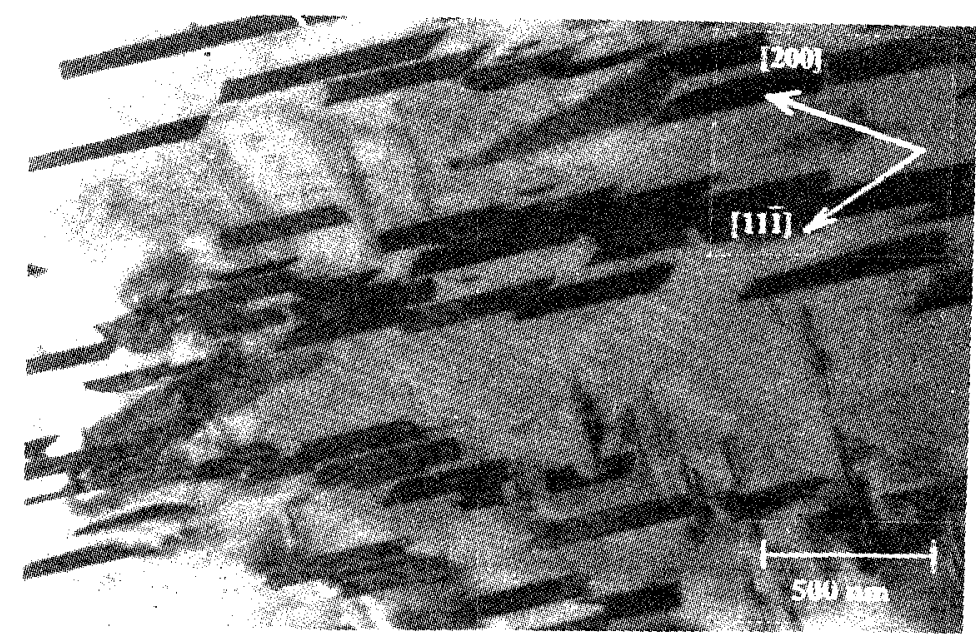

Fig. 3. TEM image of the $\Theta^{\prime}$-particles in the specimen isochronally annealed up to $533 \mathrm{~K}$.

The lifetime spectra obtained were decomposed into five components, two of them (approximately $410 \mathrm{ps}$ and $1.8 \mathrm{~ns}$ with total intensity of $\approx 6 \%$ ) being attributed to annihilations in source material. Positrons annihilating in the investigated alloy exhibited the following three lifetime components: $\tau_{1}$ varying in the range from 40 to $125 \mathrm{ps}, \tau_{2}=(207 \pm 2)$ ps and $\tau_{3}=(180 \pm 5)$ ps. Based on our results of electron microscopy and electrical resistometry and taking into account a model and data quoted in $[2,6,7]$, we suggest the following interpretation of $\tau_{2}$ and $\tau_{3}$ : $\tau_{2}$ originates from positron annihilation in the vacancies trapped at the GP zones and $\tau_{3}$ belongs to annihilations in the misfit dislocations of coherent precipitates of the $\Theta^{\prime}$-phase. The lifetime component $\tau_{1}$ apparently comes from annihilations of positrons not localized at any of the above two kinds of defects.

The dependence of the intensities of lifetime components $\tau_{2}$ and $\tau_{3}$ on the annealing temperature can be seen from Fig. 4. We interpret the first decrease in the intensity of the component $\tau_{2}$ (below $413 \mathrm{~K}$ ) by release of a part of vacancies from the GP zones during the coarsening and reorganization of the zones. These vacancies rapidly migrate and annihilate in sinks. As far as the second decrease at higher temperatures (above $473 \mathrm{~K}$ ) is concerned, we attribute it to the disappearance of the GP zones replaced by coherent precipitates of the $\Theta^{\prime}$-phase. This decrease is accompanied by appearance of lifetime component $\tau_{3}$ whose intensity increases with annealing temperature. The value of $\tau_{2}$ is in good agreement with. the results of Dlubek [2] at the beginning of the annealing sequence. The different annealing response of this component in our measurements may be attributed to a higher resolution power of our positron lifetime spectrometer. More detailed decomposition of annihilation spectra could be performed and thus the component $\tau_{3}$ was resolved in the present work.

Differential normalized annealing curves of electrical resistivity and of intensity of the lifetime component $\tau_{2}$ are plotted together in Fig. 5. Large sensitivity 


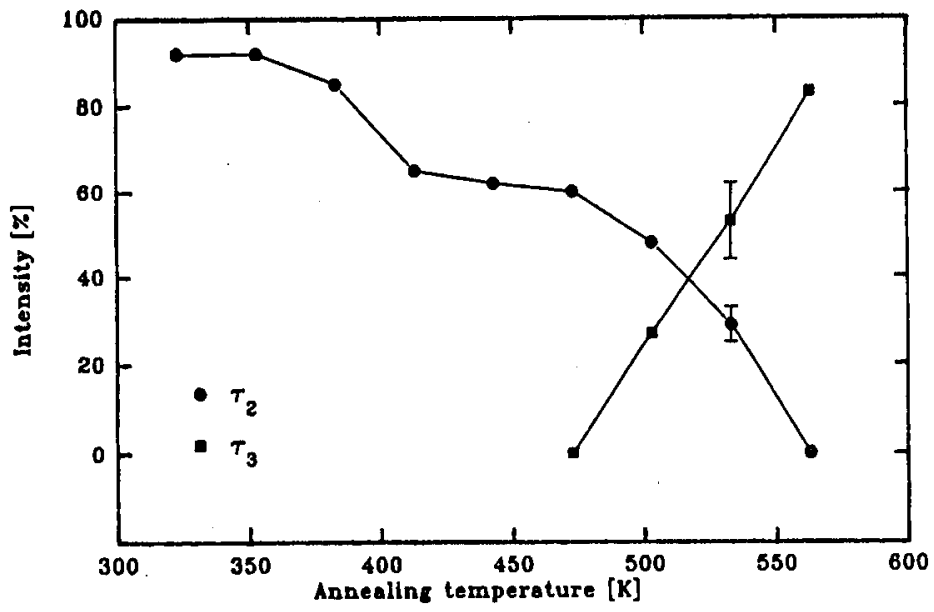

Fig. 4. Isochronal annealing response of the intensities of positron lifetime components $\tau_{2}$ and $\tau_{3}$.

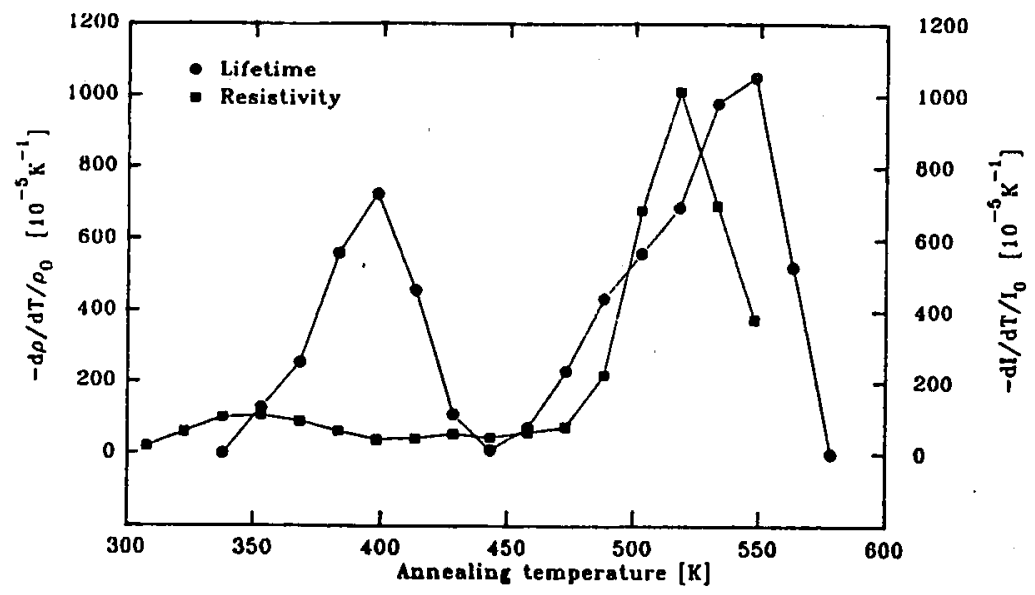

Fig. 5. Normalized differential annealing curves of the electrical resistivity and of the intensity of positron lifetime component $\tau_{2} . \varrho_{0}$ and $I_{0}$ stand for the initial values of both quantities.

of positron annihilation to the coarsening and reorganization of the GP zones in the temperature range $350 \div 450 \mathrm{~K}$ is clearly seen. Annealing response of electrical resistivity in the same temperature range is considerably less pronounced.

\section{Concluding remarks}

The combination of electrical resistivity measurements and electron microscopy with positron lifetime spectroscopy in the investigation of decomposition of 
the supersaturated Al-2 at.\% Cu alloy led to supplementary information about the behaviour of the crystal lattice defects situated at the GP zones and coherent precipitates. Moreover, the use of positron annihilation made it possible to detect separately the zones and precipitates in the temperature range where they coexist. Compared to the electrical resistometry, a more pronounced response of the positron annihilation to the coarsening and reorganization of the GP zones was found.

\section{Acknowledgment}

The present work was partly supported by the Charles University Grant No. $285 / 93$.

\section{References}

[1] P. Haasen, Physical Metallurgy, Cambridge University Press, Cambridge 1978, p. 199.

[2] G. Dlubek, Mater. Sci. Forum 13-14, 11 (1987).

[3] F. Bečváŕ, L. Lešták, I. Novotný, I. Procházka, F. Šebesta, J. Vrzal, Contrib. to the 10th Int. Conf. on Positron Annihilation, Beijing 1994, to be published in Mater. Sci. Forum.

[4] P. Kirkegaard, M. Eldrup, Comput. Phys. Commun. 7, 401 (1974).

[5] F. James, MINUIT Reference Manual, version 94.1, CERN, Geneva 1994.

[6] U.H. Gläser, G. Dlubek, R. Krause, Phys. Status Solidi B 163, 337 (1991).

[7] G. Dlubek, S. Krause, H. Krause, A.L. Beresina, V.S. Mikhalenkov, K.V. Chuistov, J. Phys., Condens. Matter 4, 6317 (1992). 